\title{
Fault line selection in cooperation with multi-mode grounding control for the floating nuclear power plant grid
}

\author{
Yikai Wang ${ }^{1}$, Xin Yin ${ }^{2 *} \mathbb{D}$, Wen $\mathrm{Xu}^{1}$, Xianggen Yin ${ }^{1}$, Minghao Wen ${ }^{1}$ and Lin Jiang ${ }^{2}$
}

\begin{abstract}
The Floating nuclear power plant grid is composed of power generation, in-station power supply and external power delivery. To ensure the safety of the nuclear island, the in-station system adopts a special power supply mode, while the external power supply needs to be adapted to different types of external systems. Because of frequent single phase-ground faults and various fault forms, the fault line selection protection should be accurate, sensitive and adaptive. This paper presents a fault line selection method in cooperation with multi-mode grounding control. Based on the maximum united energy entropy ratio (MUEER), the optimal wavelet basis function and decomposition scale are adaptively chosen, while the fault line is selected by wavelet transform modulus maxima (WTMM). For high-impedance faults (HIFs), to enlarge the fault feature, the system grounding mode can be switched by the multi-mode grounding control. Based on the characteristic of HIFs, the fault line can be selected by comparing phase differences of zero-sequence current mutation and fault phase voltage mutation before and after the fault. Simulation results using MATLAB/Simulink show the effectiveness of the proposed method in solving the protection problems.
\end{abstract}

Keywords: Floating nuclear power plant, Multi-mode grounding control, Wavelet transform modulus maxima (WTMM), Maximum united energy entropy ratio (MUEER), Phase difference, Single phase-ground fault

\section{Introduction}

The floating nuclear power plant is a moving nuclear power station, and is the combination of marine and nuclear engineering [1]. It can provide safe and effective energy supply for remote islands, offshore oil exploitation, and seawater desalination etc. It is composed of power generation, in-station power supply and external power delivery. However, the power grid can have frequent ground faults with different fault forms. Thus, it is one of the key technical challenges to quickly and accurately select and isolate the fault line under various ground fault conditions. However, conventional ground fault protection methods have low protection sensitivity

\footnotetext{
* Correspondence: xin.yin@liverpool.ac.uk

${ }^{2}$ Department of Electrical Engineering and Electronics, The University of Liverpool, Liverpool L69 3BX, UK

Full list of author information is available at the end of the article
}

under extreme fault conditions, and are difficult to adapt to different forms of ground faults. Thus, they fail to meet the requirements for safe operation of nuclear power plants.

In medium voltage (MV) shipboard systems, the shipboard hull is used as the ground loop. To ensure personnel and equipment safety in case of ground faults, ineffectively grounding modes including ungrounding, arc suppression coil grounding and resistance grounding are widely used [2]. A floating nuclear power plant grid contains sensitive loads connected with the nuclear island, which require high power supply quality. However, the neutral point ungrounding mode is prone to generating arc grounding overvoltage, and the arc suppression coil grounding method is difficult to adapt to the change of system operating status. Since the neutral point via resistance grounding method can reduce the ground 
fault current and suppress arc overvoltage through energy absorption by the grounding resistor, it has been adopted in the power grid.

At present, fault line selection algorithms mainly include intelligent [3-5], fundamental system frequency components based [6-8] and transient signal based algorithms [9-14]. The methods based on intelligent algorithms have a high ability of fault identification, but the physical meanings are not clear, and there are no unified standards for different fault forms [15]. The floating nuclear power plant system adopts the ineffectively grounding mode, the magnitude of the steady-state fault current is low [16], but there is plenty of fault information in the transient signals when ground faults occur. The transient signal based algorithms mainly include wavelet transform and traveling wave methods. Since the structure of the nuclear power plant system is compact, the fault line selection method using the traveling wave easily leads to the aliasing of the refracted and reflected traveling waves [17]. Thus, the fault features cannot be extracted effectively. In [12-14], protection methods based on WTMM have been used, in which the local characteristics of the fault signals can be effectively extracted through wavelet transform. However, the optimal wavelet transform function is usually selected through simulation, which lacks adaptive selection standards for different fault lines and fault conditions. In addition, when HIFs occur, the transient fault characteristics are not obvious so that the protection sensitivity cannot satisfy the security requirement.

Compared with common shipboard power networks and land distribution power grids, the floating nuclear power plant grid is compact in structure, and because of the ship hull being used as the ground loop, it is prone to generating different fault forms. Moreover, the reactor loads in the floating nuclear power plant are sensitive to large ground fault current. Thus, the protection method should have high sensitivity to different ground faults. To solve the problems, a protection method in cooperation with the multi-mode grounding control is proposed in this paper. When a ground fault occurs, if the fault current is larger than the threshold value, the system remains grounded with high-resistance. The protection method based on WTMM is used, and MUEER is defined to select the optimal wavelet basis and decomposition scale. When HIFs occur, the fault characteristics of the feeders are not obvious because of the signal noise and the limited precision of zero-sequence CT. Under such conditions, multi-mode grounding control is adopted and the system is switched into low-resistance grounding mode to enlarge the fault characteristic. A phase difference protection method based on zero-sequence current mutation and fault phase voltage mutation before and after the fault is applied. The performance of the proposed scheme is verified by simulation.

\section{Protection scheme in cooperation with multi- mode grounding control}

\subsection{Multi-mode grounding control}

The selection of the grounding mode of a floating nuclear power plant grid needs to consider multiple objectives such as safety of related equipments, stable system operation, and sensitive and reliable ground fault protection. The power grid of the floating nuclear power plant has a compact structure and variable operating modes. Thus, a suitable system grounding method is the key problem to ensure the safe operation of the platform.

At present, the neutral-point grounding modes of MV distribution power systems can be divided into direct grounding, ungrounding, arc suppression coil grounding and resistance grounding, etc. The MV floating nuclear power plant system has a large capacity with a large number of cable line branches, and the ship hull is directly used as the ground loop. If the ground fault current is large or the arc overvoltage is high, the safety of personnel and equipment in the plant will be compromised. Therefore, the direct grounding and ungrounding modes cannot be adopted. When the neutral-point is grounded through an arc suppression coil, it is difficult to adapt to the change of system operating status and the system can easily oscillate. Since the resistance grounding mode can reduce the fault current and suppress the arc overvoltage, it is thus adopted.

The resistance grounding mode can be divided into low-resistance, medium-resistance and high-resistance grounding. Long-term operational experience shows that if the resistance value is very low, the fault current will be large and can affect the operational safety. However, if the resistance value is very high, when HIFs occur, the fault current is small, and the line selection protection sensitivity is low, making it difficult to detect and isolate the fault accurately. Therefore, it is necessary to improve the traditional grounding mode.

Combining with the multi-resistance grounding principle [2], multi-mode grounding control is proposed. In this mode, multiple grounding resistors are installed at the neutral point of the system. Only the high-value resistor is used in normal operation. In the events of HIFs in the system, the neutral point can be switched into low-resistance mode by multi-mode grounding control, to amplify the fault characteristic, and improve the reliability of line selection protection. The structure of the floating nuclear power plant system is shown in Fig. 1.

As shown in Fig. 1, the system consists of a generator, a neutral multi-mode grounding device and four branch lines, while the power source and the network are 


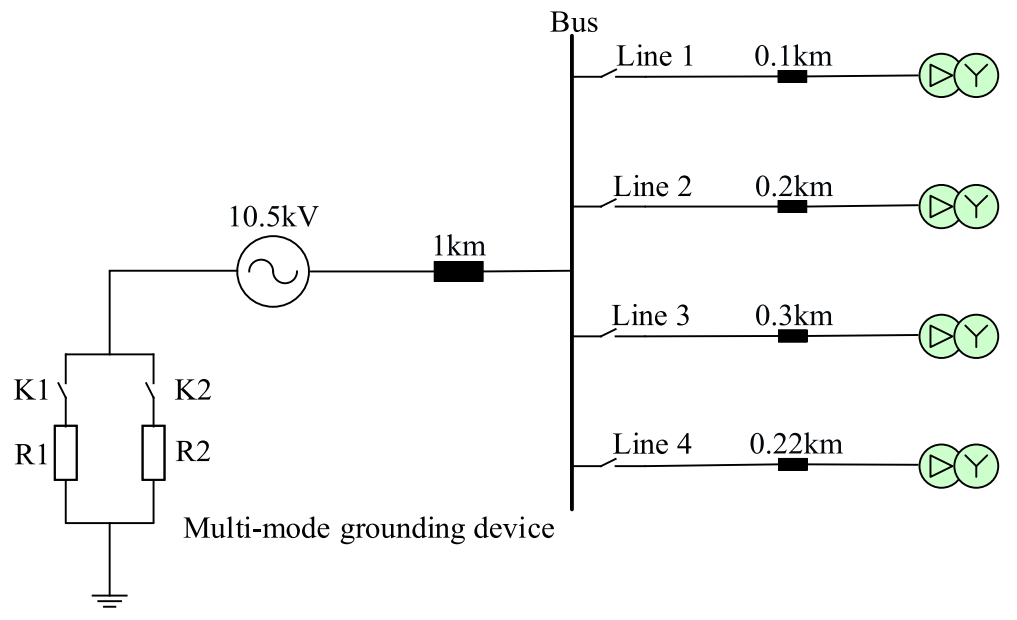

Fig. 1 Floating nuclear power plant MV system

directly connected. The ends of each line is connected to the in-station or external system through a separate transformer. In normal operation, switch $\mathrm{K} 1$ is connected and $\mathrm{K} 2$ is disconnected, and the system is grounded by the high-resistance R1. This can effectively reduce the fault current in the cases of metal and lowimpedance ground faults, and suppress the transient overvoltage in the cases of intermittent arc ground faults. When the zero-sequence fault current magnitude meets the threshold value, the fault line can be selected based on the WTMM protection method. When HIFs occur, the fault current magnitude is low, which makes it difficult for the measuring devices to meet the precision requirements. Under such conditions, switch $\mathrm{K} 2$ is closed by the multi-mode grounding control and the system is switched into low-resistance mode to increase the fault current. In addition, the line selection protection method for HIFs is used to select the ground fault line. In this way, the sensitivity of the protection scheme can be improved and the safety of the floating nuclear power plant can be assured.

\subsection{Coordination of the protection method and neutral- point grounding mode}

During normal operation state, the high-resistance grounding mode is adopted. When metal, low-impedance or intermittent arc ground faults occur, the magnitude of the fault current transient component is relatively large, and satisfies the threshold value requirement. MUEER is used to select the optimal wavelet transform function and decomposition scale, and WTMM is used to select the fault line. By comparing the WTMM polarity and square value differences of the zero-sequence current in the lines, the fault line can be quickly and accurately selected.

When HIFs occur, the fault characteristic is not obvious. Since the precision of the zero-sequence CT is limited and the fault characteristic signal is greatly affected by interference signals, it is difficult to select the fault line correctly. In cooperation with the multi-mode grounding control, the neutral-point can be switched into low-resistance mode to amplify the fault characteristic. In this situation, the voltages of the sound phases are almost unchanged. Thus, the fault line can be selected by comparing the phase differences of the zerosequence current mutation and fault-phase voltage mutation before and after the fault between the lines. The coordination strategy of the multi-mode grounding control and the fault line selection scheme is shown in Fig. 2.

\section{Line selection protection method based on WTMM}

The floating nuclear power plant grid is a system with multi branches and short cable lines. Since the system is grounded by high-resistance in the normal state, a large number of transient fault components will be generated when ground faults occur. Except for extreme HIFs, the transient fault current can meet the threshold value requirement. In order to make good use of the local fault characteristics of the transient signals, an adaptive protection method based on WTMM is proposed.

\subsection{Principle of wavelet transform singularity detection}

If a function $f(x)$ is discontinued somewhere on the domain, or its derivative is not continuous, it is regarded as singular. Whether the function $f(x)$ is singular can be characterized by the Lipschitz function and the function can also be used for characterization of the signal singularity detection [18]. This refers to the detection and extraction of signal mutation points. Where $n$ is a nonnegative integer and $a$ satisfies $n \leq a \leq n+1$, if there are two constants $\mathrm{A}, h_{0}>0$, and an n-degree polynomial 


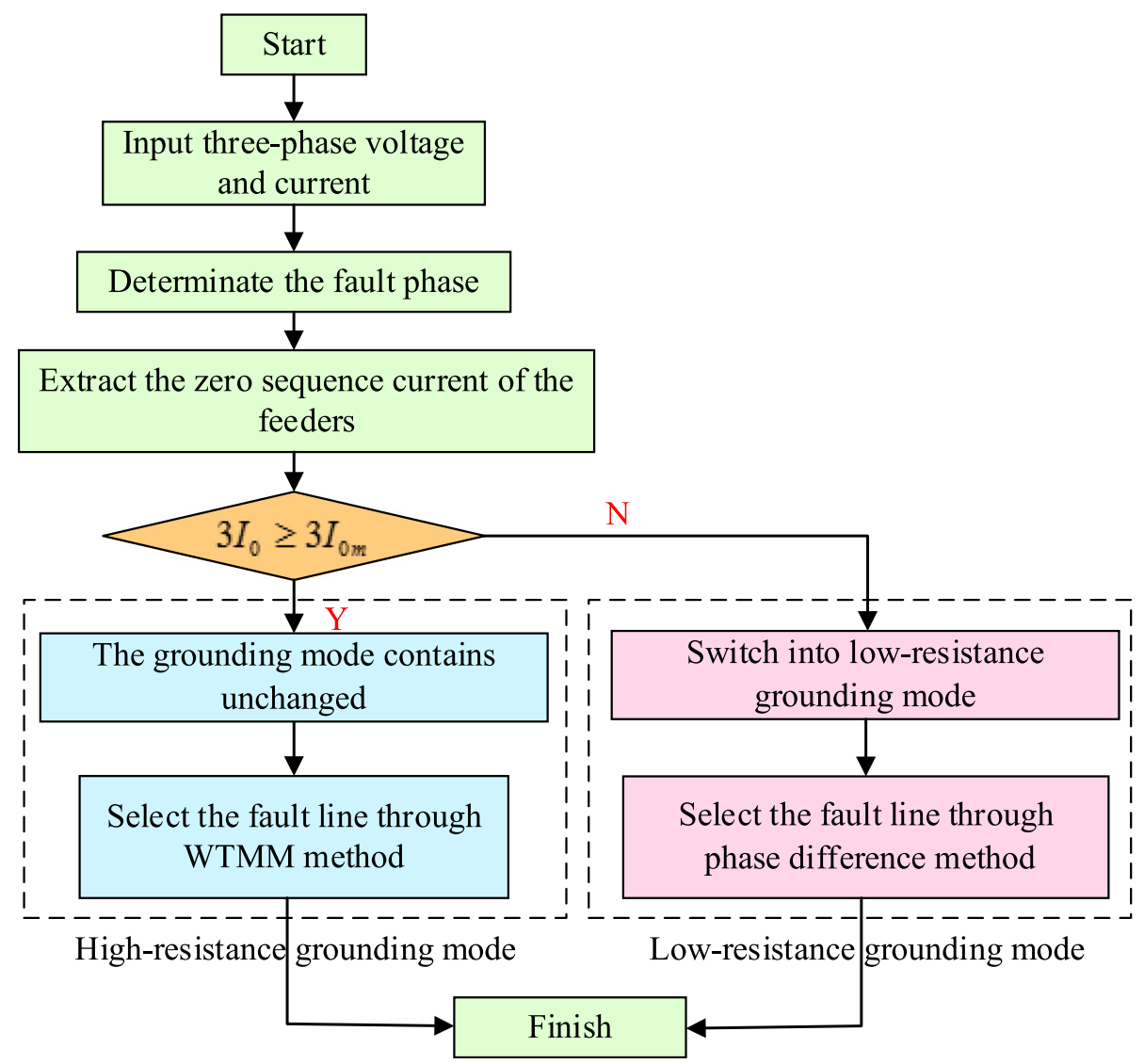

Fig. 2 Flowchart of the fault line selection scheme

$P_{n}(x)$, for any $h \in\left[-h_{0}, h_{0}\right]$, the following can be established:

$$
\left|f\left(x_{0}+h\right)-P_{n}(h)\right| \leq A|h|^{\alpha}
$$

Then the function $f(x)$ is taken to be Lipschitz at the point $x_{0}$. The Lipschitz property of $f(x)$ at point $x_{0}$ indicates the singularity degree of the function at that point. The larger $a$ is, the smoother the function $f(x)$ is at that point, whereas the smaller $a$ is, the more singular the function $f(x)$ is. The singularity of the function $f(x)$ can be characterized by its WTMM. The theory shows that the magnitude of the WTMM represents the strength of the signal mutation, and the polarity represents the direction of the signal mutation.

When ground faults occur in the nuclear power plant system, the transient fault components at the initial stage are all abrupt or singular. According to the principle of wavelet transform singularity detection, the singularity of the WTMM of the transient zerosequence current corresponds to the original fault signals. The transient fault current of each line can be decomposed by wavelets, and then the WTMM can be solved after wavelet reconstruction. By comparing the polarities of the WTMM, it can determine whether the fault occurs on the bus or on the lines. For line faults, the line with the largest square value of the WTMM is regarded as the fault line.

\subsection{Optimal wavelet basis and decomposition scale selection method based on MUEER}

The oprational environment of the floating nuclear power plant is complex, and various ground fault forms can occur. Wavelet transform can effectively extract the local characteristics of the transient fault current and has high accuracy in fault line selection. However, if the wavelet basis and decomposition scale are selected improperly, the WTMM cannot effectively reflect the fault characteristics, leading to misjudgment and affecting the safety of the system. Therefore, the corresponding evaluation criteria is formulated to select the best wavelet basis and decomposition scale of the fault signals. Based on the energy of the wavelet transform results, and the correlation between wavelet basis functions and fault transient signals, a fusion index which can guide the optimal wavelet basis and decomposition scale for fault line selection is proposed. 
Performing wavelet decomposition on the fault signal $Y(i)$ through the wavelet basis $d b(k)$ and decomposition scale $j$ with the sampling length of $N$ to obtain the wavelet coefficient $W(j, k)$. Its energy $E$ can be described as

$$
E_{j, k}=\sum_{i=1}^{N}|W(j, k)|^{2}
$$

According to the Parserval theorem [19], the energy of the fault signal after orthogonal wavelet transform is equal to its origin. As WTMM is used to select the fault line, larger wavelet coefficient energy makes it easier to select the fault line accurately. Therefore, the energy value of the wavelet coefficient is used as an indicator of the energy of the wavelet transform.

The essence of the wavelet transform method is to extract local fault information from transient fault signals. When the center frequencies of the wavelet basis function and the transient fault signal are close, the wavelet transform results can effectively reflect the fault information. The concept of cross entropy in information theory is introduced to measure the correlation of the center frequency between transient fault signals and wavelet basis functions [20]. It can be used to describe the difference between two probability distributions. The expression of the cross entropy between $X$ and $Y$ can be described as

$$
H(X, Y)=-\sum_{x \in X} \sum_{y \in Y} p(x, y) \log p(x, y)
$$

In this paper, the energy distribution perspective is used to define the two-dimensional cross entropy between the fault signal and wavelet basis function. The energy distribution probability of the wavelet basis function $d b(k)$ can be described as

$$
p(d b)=\frac{|d b(k)|^{2}}{\sum_{j=1}^{k}|d b(k)|^{2}}
$$

The energy distribution probability of the fault signal $Y(i)$ can be described as

$$
p(Y)=\frac{|Y(i)|^{2}}{\sum_{i=1}^{N}|Y(i)|^{2}}
$$

The joint energy distribution probability of the fault signal $Y(i)$ and the wavelet basis $d b(k)$ can be defined as

$$
p\left(Y_{i}, d b_{k}\right)=\frac{|Y(i)|^{2}|d b(k)|^{2}}{\sum_{i=1}^{N} \sum_{j=1}^{k}|Y(i)|^{2}|d b(k)|^{2}}
$$

Then the cross entropy can be defined as

$$
H\left(Y_{i}, d b_{k}\right)=-\sum_{Y} \sum_{d b} p\left(Y_{i}, d b_{k}\right) \log p\left(Y_{i}, d b_{k}\right)
$$

In fault line selection, the smaller the cross entropy, the higher the degree of fit of the wavelet basis function to the original fault signal and the more effective information can be extracted. Therefore, the cross entropy can be used as an indicator of the center frequency correlation between the wavelet basis functions and the fault signals.

In order to select the optimal wavelet basis function and decomposition scale, based on the cross entropy and the wavelet transform energy, a fault line selection method based on the united energy entropy ratio is proposed, it is

$$
R=\frac{E_{j, k}}{H\left(Y_{i}, d b_{k}\right)}
$$

A larger $R$ indicates larger correspondence of the wavelet coefficient amplitude to the fault signal, higher degree of similarity with the original fault signal, and better reflection of the transient local characteristics of the fault signal. Therefore, the optimal wavelet basis function and decomposition scale can be selected by comparing the values of $R$.

\subsection{Fault line selection method based on WTMM}

The wavelet transform coefficient represents the frequency component of the fault signal at a certain decomposition scale. The magnitude of the WTMM indicates the mutation strength and the polarity represents the mutation direction.

The WTMM value of the zero-sequence current in the fault line is the largest and its polarity is opposite to other lines. This characteristic can be used to construct a protection criterion to distinguish bus and feeder faults. The fault line selection protection process based on WTMM is shown in Fig. 3.

After detecting the ground fault in the power system, the optimal wavelet basis function and decomposition scale of the transient zero-sequence fault current are selected by MUEER. The optimal wavelet basis function is used to perform wavelet decomposition and reconstruction of the transient zero-sequence current of each line, and the magnitude and polarity of the WTMM of the zero-sequence current of each line after the reconstruction are solved. By comparing the polarities of the WTMM, it can determine whether the fault occurs on 


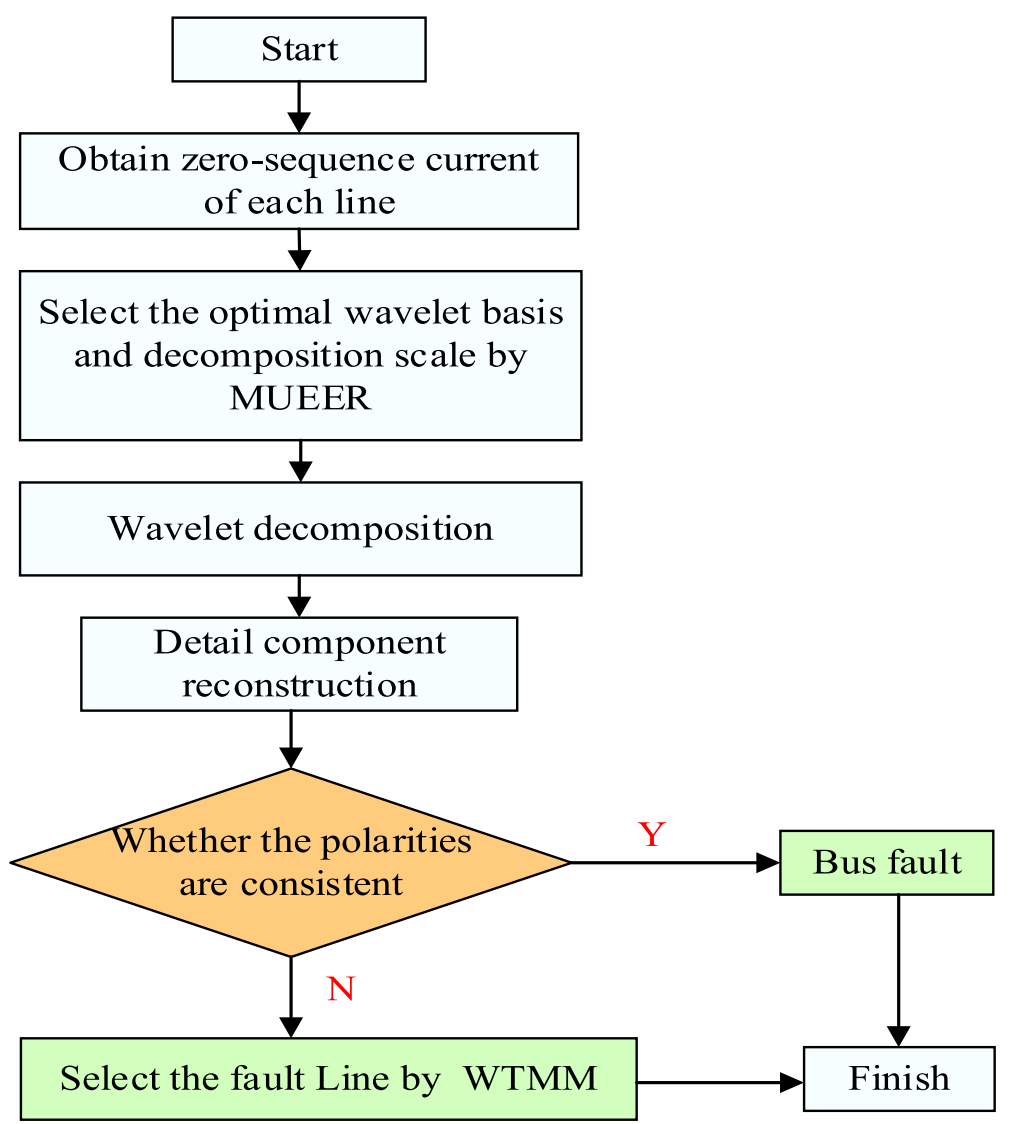

Fig. 3 Flow chart of WTMM protection method

the bus or the feeder. If the polarities are the same, the fault is on the bus, otherwise it is on the feeder.

The fault line selection method based on WTMM uses the transient fault signals to realize the protection function. For intermittent arc light grounding faults, the transient process is intense, and the amplitudes of the WTMM are large for all the lines. However, for lowimpedance grounding faults, the WTMM amplitude of the fault line is usually larger than 1, while the WTMM amplitude is usually less than 1 . Taking the square value of the WTMM amplitude can further enlarge the difference between the fault and the sound lines, so as to improve the protection sensitivity. In this way, the line with the largest square value of the WTMM is finally regarded as the fault line.

\section{Line selection protection method for HIFs based on phase difference}

The floating nuclear power plant grid is prone to HIFs because of its compact construction and the use of the shipboard hull as the ground loop. When HIFs occur, the fault current is small and the line selection protection sensitivity is low, making it difficult to detect and remove the fault accurately.
To improve the sensitivity and accuracy of the protection, the line selection scheme is combined with the system grounding mode. In cooperation with the multimode grounding control, when HIFs occur, the system is switched into low-resistance grounding mode to enlarge the fault current. In this operational mode, the voltage of the non-fault phase is nearly unchanged. Based on this, a protection method for HIFs is proposed.

Figure 4 illustrates a simplified circuit diagram during HIFs. As shown, when HIFs occur, the ground fault current can be obtained by

$$
\dot{I}_{f}=\dot{I}_{d}+\dot{I}_{c}
$$

where $\dot{I}_{d}$ is the current through the neutral-point and $\dot{I}_{c}$ is the capacitance current to the ground. Since the neutral-point voltage is equal to the zero-sequence voltage which is equal everywhere, the fault current can be obtained as

$$
\dot{I}_{d}=\frac{\dot{U}_{0}}{R_{d}} \dot{I}_{c}=j 3 \omega C_{0 \Sigma} \dot{U}_{0}
$$

where $U_{0}$ is the zero-sequence voltage in the system, $R_{d}$ is the neutral-point grounding resistance and $C_{0 \Sigma}$ is the 


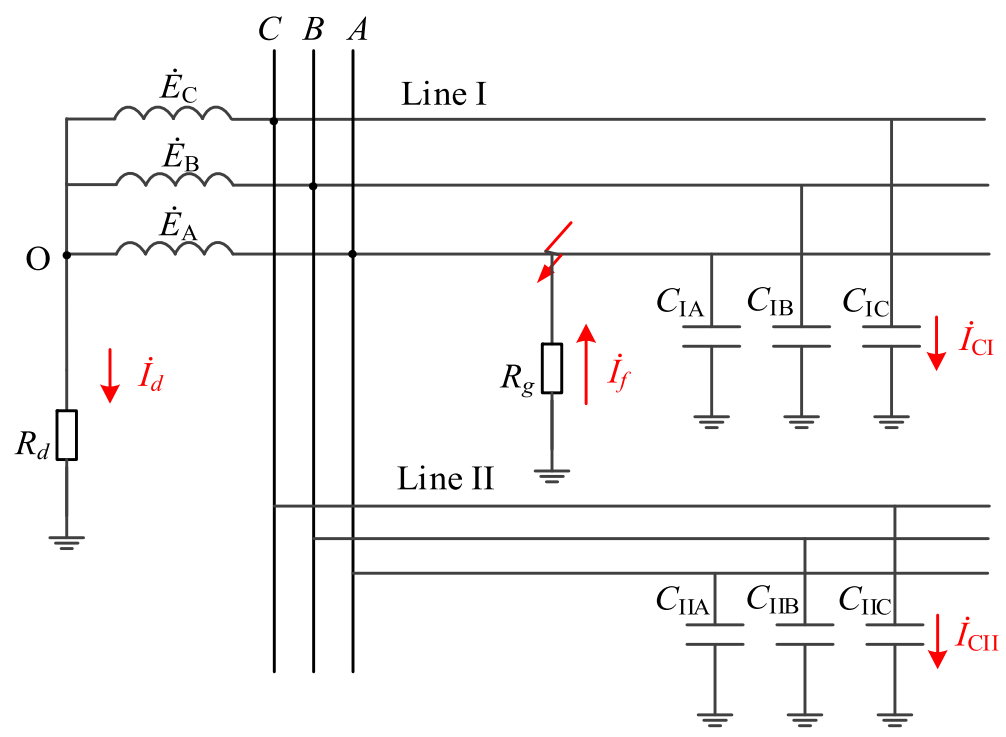

Fig. $4 \mathrm{HIF}$ in low-resistance grounding system

sum of the capacitance to ground. Assuming that the HIF occurs on phase A in line I, the fault point can be considered as a superimposed zero-sequence voltage source. Thus, the ground fault current can be calculated by

$$
\dot{I}_{f}=-\frac{\dot{U}_{0}+\dot{U}_{A}}{R_{g}}
$$

where $U_{A}$ is the phase voltage before the fault and $R_{g}$ is the transition ground fault impedance. Substituting ( 9 , 10) into (11), the zero-sequence voltage of the system can be obtained as

$$
\dot{U}_{0}=\frac{-\dot{U}_{A}}{1+\frac{R_{g}}{R_{d}}+j 3 \omega C_{0 \Sigma} R_{g}}
$$

In this system, after the occurrence of an HIF, the zero-sequence voltage is superimposed on the original non-fault phase voltage. Thus, the modulus of the zero-sequence voltage is the magnitude mutation of the non-fault phase voltage.

For HIFs, compared to $R_{g}, R_{d}$ is very small. Thus, the magnitude of the zero-sequence voltage is also small. Therefore, it can be considered that the voltage of the non-fault phase remains unchanged in the process of the fault.

In Fig. 4, before the fault, the zero-sequence current for fault line I can be calculated by

$$
\dot{I}_{\mathrm{I} 0}=\dot{U}_{A} j \omega C_{\mathrm{I} A}+\dot{U}_{B} j \omega C_{\mathrm{I} B}+\dot{U}_{C} j \omega C_{\mathrm{I} B}
$$

After the fault, the zero-sequence current can be calculated by

$$
\dot{I}_{\mathrm{I} 0}{ }^{\prime}=\dot{U}_{A}{ }^{\prime}\left(j \omega C_{\mathrm{I} A}+\frac{1}{R_{g}}\right)+\dot{U}_{B}{ }^{\prime} j \omega C_{\mathrm{I} B}+\dot{U}_{C}{ }^{\prime} j \omega C_{\mathrm{I} C}
$$

Subtracting (13) from (14), the zero-sequence current mutation of the fault line before and after the fault can be obtained as

$$
\Delta \dot{I}_{\mathrm{I} 0}=\Delta \dot{U}_{A} j \omega C_{\mathrm{I} A}+\frac{\dot{U}_{A}{ }^{\prime}}{R_{g}}
$$

Similarly, for the sound line II, the zero-sequence current mutation before and after the fault can be obtained as

$$
\Delta \dot{I}_{\mathrm{II} 0}=\Delta \dot{U}_{A} j \omega C_{\mathrm{II} A}
$$

Based on the theoretical analysis above, the phasordiagram of the system under HIFs can be drawn as Fig. 5.

For the sound line II, the phase difference between $\Delta$ $\dot{U}_{A}$ and $\Delta \dot{I I I O}_{\mathrm{II}}$ is $90^{\circ}$ as seen from (16) and Fig. 5. However, for the fault line I, as shown in (15) and Fig. 5, the fault current is affected by the system capacitance to ground $C_{0 \Sigma}$, neutral ground resistance $R_{d}$ and ground transition resistance $R_{g}$, and the phase difference of $\Delta \dot{I}_{\text {I0 }}$ and $\Delta \dot{U}_{A}$ is around $180^{\circ}$. Thus, combining the theoretical derivation and simulation results, the fault line selection protection criterion based on phase difference can be constructed as

$$
120^{\circ}<\arg \left(\frac{\Delta \dot{I}_{i 0}}{\left.\Delta \dot{U}_{\phi}\right)<240^{\circ}}\right.
$$

where $\Delta \dot{I}_{i 0}$ is the zero-sequence current mutation in 


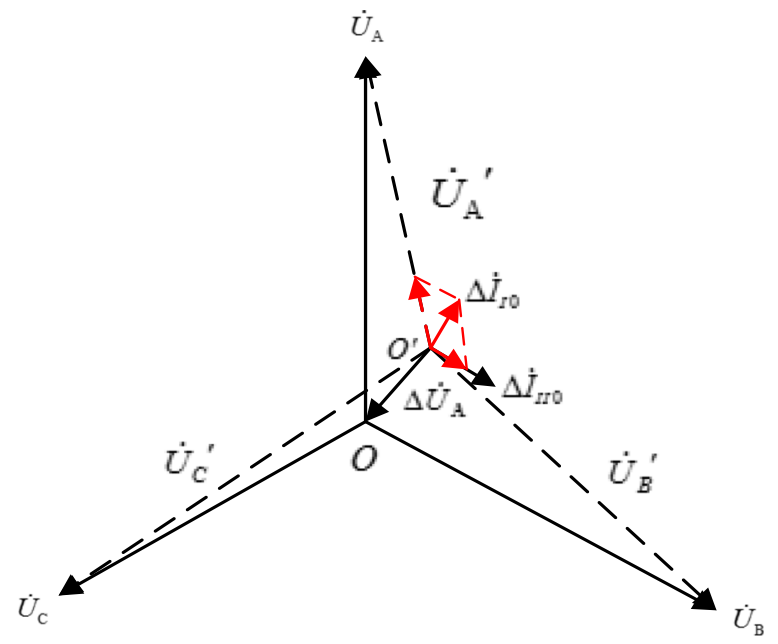

Fig. 5 Phasor-diagram of the system under HIFs

each line $(i=1,2,3, \ldots)$ and $\Delta \dot{U}_{\phi}$ is the mutation of the fault phase voltage $(\phi=a, b, c)$. However, the phase difference is influenced by network parameters and fault modes etc. Thus, certain margins have been included to improve the protection sensitivity.

After detecting the ground fault in the power system, if the zero-sequence current magnitude is small and does not reach the threshold value, it can be determined as an HIF. In cooperation with the multi-mode grounding control, the neutral point is switched into lowresistance mode to enlarge the zero-sequence current. By comparing the phase difference of the zero-sequence current mutation and the fault phase voltage mutation of each line before and after the fault, the fault line can be selected.

\section{Simulation results and discussion}

MATLAB/Simulink software is used to establish the equivalent model of the MV power system of the floating nuclear power plant shown in Fig. 1 and implement the algorithms. During normal operation, the system operates in high-resistance grounding mode. When a ground fault occurs, the WTMM protection method is used to select the fault line. When an HIF occurs, the system is switched into low-resistance mode by the multi-mode grounding control and the phase difference method based on the HIF characteristic is used. The MV system includes 4 main cables, each of which has multiple branches but made equivalent to one single line in order to simplify the calculation. The length of each line has been marked in Fig. 1.

\subsection{Simulation analysis of WTMM protection method}

In the floating nuclear power plant system shown in Fig. 1 , the ground fault is set at the end of line 3. The fault forms include a metal ground fault (Fault 1), a lowimpedance ground fault (with a transition ground fault impedance of $50 \Omega$ ) (Fault 2) and an intermittent arc ground fault (Fault 3). In the simulation process, the intermittent arc ground fault model is established based on the principle of fundamental frequency arc extinguishing. After the zero-sequence current signals of each line are obtained, the optimal wavelet basis function and decomposition scale can be selected based on MUEER. As for $d b(N)$ wavelet basis, its support length, regularity and vanishing moment increase with the value of $N$. Since the above characters are mutually limited, it has a good decomposition effect and has been commonly used in fault line selection. Thus, the optimal wavelet basis is selected from $d b$ wavelet basis functions, and then the fault signals are decomposed and reconstructed through the optimal wavelet basis function and decomposition scale to obtain the magnitude and polarity of WTMM of each line. The simulation results of the above ground fault forms are shown in Table 1.

Take the intermittent arc ground fault (fault 3) as an example. Because of the local high-frequency transient information in the zero-sequence fault current, a wavelet basis function with a higher vanishing moment needs to be selected for decomposition. The simulation results show that the optimal wavelet basis of the lines is $d b 10$ and the optimal decomposition scale parameter is the 9th dimension. Since the polarity of the WTMM of line

Table 1 Simulation results based on WTMM

\begin{tabular}{|c|c|c|c|c|}
\hline \multicolumn{2}{|c|}{$\begin{array}{l}\text { Fault forms } \\
\text { Results }\end{array}$} & \multirow{2}{*}{$\begin{array}{l}\text { Fault } 1 \\
d b 3\end{array}$} & \multirow{2}{*}{$\begin{array}{l}\text { Fault } 2 \\
d b 3\end{array}$} & \multirow{2}{*}{$\begin{array}{l}\text { Fault } 3 \\
d b 10\end{array}$} \\
\hline Line 1 & Optimal function & & & \\
\hline & Optimal scale & 5 & 5 & 9 \\
\hline & Square value & 0.0758 & 0.0753 & 20.0580 \\
\hline & Polarity & + & + & + \\
\hline \multirow[t]{4}{*}{ Line 2} & Optimal function & $d b 3$ & $d b 3$ & $d b 10$ \\
\hline & Optimal scale & 5 & 5 & 9 \\
\hline & Square value & 0.3034 & 0.3014 & 74.8303 \\
\hline & Polarity & + & + & + \\
\hline \multirow[t]{4}{*}{ Line 3} & Optimal function & $d b 3$ & $d b 3$ & $d b 10$ \\
\hline & Optimal scale & 5 & 4 & 9 \\
\hline & Square value & 40.2709 & 35.5729 & 4407.7 \\
\hline & Polarity & - & - & - \\
\hline \multirow[t]{4}{*}{ Line 4} & Optimal function & $d b 3$ & $d b 3$ & $d b 10$ \\
\hline & Optimal scale & 5 & 5 & 9 \\
\hline & Square value & 0.3671 & 0.3647 & 93.2945 \\
\hline & Polarity & + & + & + \\
\hline \multicolumn{2}{|c|}{ Selection result } & Line3 & Line3 & Line3 \\
\hline \multicolumn{2}{|c|}{ Whether correct } & Correct & Correct & Correct \\
\hline
\end{tabular}


3 is opposite to the others, and its square value of the WTMM is the largest, it is judged as the fault line.

The results of other ground fault conditions are listed in Table 1. It can be seen that for different fault conditions and fault lines, the optimal wavelet basis functions and the decomposition scales are different. Based on the MUEER method, the cross entropy and the wavelet transform energy are used to effectively and adaptively select the optimal wavelet basis function and decomposition scale parameters of the zero-sequence currents in each line. The simulation results show that the protection method based on WTMM can reflect the local fault characteristic, and it adapts to various ground fault forms which can occur in the floating nuclear power plant power system.

\subsection{Simulation analysis of phase difference protection}

For HIFs, the faults are set at the end of the lines and at the bus, and the value of the transition ground fault impedance is $5 \mathrm{k} \Omega$. Since the zero-sequence current of each line is small and does not reach the threshold value, the neutral-point is switched into low-resistance grounding mode by the multi-mode grounding control. According to [21], the resistance value in the low-resistance grounding mode of the MV power system can be calculated by the capacitance parameters of the power grid. Assuming a metal ground fault occurs in the system, and the resistance value can be obtained by making the capacitance current equal to the current through the neutral grounding resistance. The phase differences of the zero-sequence fault current mutation and fault phase voltage mutation of each line before and after the fault are shown in Table 2.

According to the simulation results, when HIFs occur, the phase differences between the zero-sequence current mutation and the fault phase voltage mutation of the sound lines are around $90^{\circ}$. For the fault line, the phase difference closes to $180^{\circ}$, which meets the protection criterion. When HIFs occur at the bus, the phase differences of all the lines are around $90^{\circ}$. In this way, ground faults of the bus and the feeders can be effectively distinguished.

Table 2 Simulation results under HIFs

\begin{tabular}{llllll}
\hline $\begin{array}{l}\text { Fault Line } \\
\text { Results }\end{array}$ & 1 & 2 & 3 & 4 & Bus \\
\hline Line 1 & $179.2^{\circ}$ & $89.5^{\circ}$ & $89.6^{\circ}$ & $89.6^{\circ}$ & $89.6^{\circ}$ \\
Line 2 & $87.4^{\circ}$ & $179.2^{\circ}$ & $87.4^{\circ}$ & $87.4^{\circ}$ & $87.4^{\circ}$ \\
Line 3 & $89.6^{\circ}$ & $89.5^{\circ}$ & $179.2^{\circ}$ & $89.6^{\circ}$ & $89.6^{\circ}$ \\
Line 4 & $89.6^{\circ}$ & $89.5^{\circ}$ & $89.6^{\circ}$ & $179.2^{\circ}$ & $89.6^{\circ}$ \\
Selection result & Line 1 & Line 2 & Line 3 & Line 4 & Bus \\
Whether correct & Correct & Correct & Correct & Correct & Correct \\
\hline
\end{tabular}

In cooperation with the system grounding mode, this protection method can effectively increase the fault characteristic quantity when HIFs occur in the system, in order to select the fault line based on the electrical quantity change characteristics of HIFs. It can accurately distinguish between bus and feeder faults, has a higher resistant ability to high transition ground fault impedance, and improves the sensitivity of line selection protection in the case of HIFs in floating nuclear power plant power systems.

\section{Conclusion}

In this paper, a ground fault protection scheme for the floating nuclear power plant system in cooperation with multi-mode grounding control is proposed. Theoretical and simulation analysis have shown that:

1) Through multi-mode grounding control, the protection scheme is effectively combined with the system grounding mode to improve the sensitivity and accuracy of the fault line selection protection.

2) In the protection method based on WTMM, MUEER is defined to adaptively select the optimal wavelet basis function and decomposition scale. This method can be effectively applied under metal, low-impedance and intermittent arc ground faults;

3) In cooperation with the multi-mode grounding control, a phase difference protection criterion based on the HIFs characteristic is proposed to effectively increase the fault characteristic quantity leading to high sensitivity in extreme HIF situations;

4) The MV power system of the floating nuclear power plant is taken as an example to analyze and illustrate the protection method. The proposed method is also applicable to the in-station and external power systems.

Abbreviations

WTMM: Wavelet transform modulus maxima; MUEER: Maximum united energy entropy ratio; MV: Medium voltage

Acknowledgements

Not applicable.

Authors' contributions

XGY participated in the simulation model building. YKW, XY and WX drafted the manuscript. YKW and XY carried out the method of WTMM fault line selection protection method. MHW and $\amalg$ carried out the phase difference fault line selection protection method. The authors read and approved the final manuscript.

\footnotetext{
Author's information

Yikai Wang, male, received the B.S. degree from North China Electric Power University, he is currently working toward the Ph.D. degree at Huazhong University of Science and Technology, major in power system protection and control.

Xin Yin, male, IEEE member, received the Ph.D. degree from the University of Manchester, U.K.. He is presently working as a Postdoctoral Research Associate of electrical engineering with the University of Liverpool. His
} 
current research interests include distribution system and control of micro grid with renewable energy.

Wu Xen, female, received the B.S. degree from Tsinghua University, she is currently working toward the M.Sc. degree at Huazhong University of Science and Technology, major in power system protection and control. Xianggen Yin, male, IEEE member, PHD and Professor, major in protective relaying and power system stability control.

Minghao Wen, male, IEEE member, PHD and Professor, major in protective relaying and power system stability control.

Lin Jiang, male, IEEE member, received the Ph.D. degree from the University of Liverpool, U.K.. He is presently working as a Reader of electrical engineering with the University of Liverpool. His current research interests include the optimization and control of smart grids, electrical machines, power electronics, and renewable energy.

\section{Funding}

Project Supported by National Natural Science Foundation of China (No.51877089). Research on the mechanism and fault ride-through integrated strategies of an active power router in hybrid AC and DC distribution grids.

\section{Availability of data and materials}

The datasets used during the current study are available from the corresponding author on reasonable request.

\section{Competing interests}

The authors declare that they have no competing interests.

\section{Author details}

'State Key Laboratory of Advanced Electromagnetic Engineering and Technology, Huazhong University of Science and Technology, Wuhan 430074, Hubei, China. ${ }^{2}$ Department of Electrical Engineering and Electronics, The University of Liverpool, Liverpool L69 3BX, UK.

Received: 20 March 2020 Accepted: 25 May 2020

Published online: 01 July 2020

\section{References}

1. Li, Z. H. A. N. G., Zhoufeng, L. I. U., Bocai, D. I. N. G., et al. (2017). Design essentials of power system at offshore nuclear power platform[J]. Marine Electric \& Electronic Engineering, 37(11), 36-37.

2. Yunchao, W. A. N. G., Zhanqing, Y. U., Jinliang, H. E., et al. (2015). Performance of shipboard medium-voltage DC system of various grounding modes under monopole ground fault[J]. IEEE Transactions on Industry Applications, 51(6), 5002-5009.

3. Yunfeng, X. I. O. N. G., Shisheng, W. A. N. G., \& Wenqian, Z. H. A. N. G. (2012). A line-selection method of data fusion based on neural network[J]. International Workshop on Information and Electronics Engineering, 29, 520-525.

4. Anbo, M. E. N. G., Jiafei, G. E., Deqiang, L. I., et al. (2016). Research on fault line selection of distribution network using RBF neural network based on crisscross optimization algorithm optimization[J]. Power System Protection and Control, 44(21), 90-95.

5. Geethanjali, M., \& Priya, K. S. (2009). Combined wavelet transforms and neural network (WNN) based fault detection and classification in transmission lines[C]. In Proceedings of International conference on control, automation, communication and energy conservation, Kongu Engn Coll, Perundurai, 4-6 June (pp. 1-7).

6. Zheng, Q. I. (2005). Research on technology of line detection and fault location for single-phase-to-earth in neutral point non-effectively grounded systems[D]. Beijing: North China Electric Power University.

7. Aucion, B. M., \& Jones, R. H. (1996). High impedance fault detection implementation issues. IEEE Transactions on Power Delivery, 11(1), 212-217.

8. Zhichao, L. I. N., Xinxing, L. I. U., Yingmin, W. A. N. G., et al. (2018). Grounding fault protection based on zero sequence current comparison in low resistance grounding system[J]. Power System Protection and Control, $46(22), 15-21$.

9. Rui, L. I. A. N. G., Lianhua, C. U. I., Zhili, D. U., et al. (2014). Fault line selection and location in distribution power network based on traveling wave time difference of arrival relationships[]]. High Voltage Engineering, 40(11), 3411-3417.

10. Zhiyong, L. I., Wei, W. A. N. G., Liangwen, Z. H. U. A. N. G., et al. (2019). Dynamic test of traveling wave line selecting device based on RTDS small time-step simulation[J]. Power System Protection and Control, 47(23), 108-114.
11. Mingming, X. U., Shuping, G. A. O., Zhongxue, C. H. A. N. G., et al. (2018) Model recognition based single-phase earthed faulty line selection method of Petersen coil grounded system[J]. Power System Protection and Control, 46(2), 73-78.

12. Xinzhou, D. O. N. G., \& Shenxing, S. H. I. (2008). Identifying single-phase-to-ground fault feeder in neutral noneffectively grounded distribution system using wavelet transform[J]. IEEE Transactions on Power Delivery, 23(4), 1829-1837.

13. Sedighi, A. R., Haghifam, M. R., Malik, O. P., et al. (2005). High impedance fault detection based on wavelet transform and statistical pattern recognition[J]. IEEE Transactions on Power Delivery, 20(4), 2414-2421.

14. MICHALIK, M., REBIZANT, W., LUKOWICZ, M., et al. (2006). High impedance fault detection in distribution networks with use of wavelet based algorithm[J]. IEEE Transactions on Power Delivery, 21(4), 1793-1802.

15. Bin, W. A. N. G., Xin, C. U. I., \& Xinzhou, D. O. N. G. (2020). Overview of arc high impedance grounding fault detection technologies in distribution system[J]. Proceedings of the CSEE, 40(1), 96-107 377.

16. Baohui, Z. H. A. N. G., Zhiguo, H. A. O., \& Zhiqian, B. O. (2016). New development in relay protection for smart grid[]]. Protection and Control of Modern Power Systems, 1(2), 121-127. https://doi.org/10.1186/s41601-0160025-x.

17. Zhiwei, C. H. E. N., \& Qin, S. H. U. (2015). Study on single phase ground fault location of mixed distribution network based on neural network and SVD[J]. Digital Technology and Application, 11, 100-103.

18. Bianling, S. U. (2016). Application of wavelet transform in power system[M] (pp. 39-42). Beijing: Science Press.

19. Quan, P. A. N. G., Cuirong, Y. A. N. G., Yingle, F. A. N., et al. (2007). Image segmentation based on the local minimum cross-entropy and quadtree[C]. In Proceedings of 5th International Conference on Wavelet Analysis and Pattern Recognition, Beijing, China (pp. 356-359).

20. Ray, P., Mishra, D. P., \& Mohaptra, S. (2016). Fault classification of a transmission line using wavelet transform \& fuzzy logic[C]. In Proceedings of 1st IEEE International Conference on Power Electronics, Intelligent Control, and Energy Systems (ICPEICES), Delhi, India, (pp. 1-6).

21. Xiaoqi, F. U. (2014). Research on the technology and application of $10 \mathrm{kV}$ distribution network neutral grounding through small resistance[D]. Beijing: North China Electric Power University.

\section{Submit your manuscript to a SpringerOpen ${ }^{\circ}$ journal and benefit from:}

- Convenient online submission

- Rigorous peer review

- Open access: articles freely available online

High visibility within the field

- Retaining the copyright to your article

Submit your next manuscript at $\boldsymbol{\nabla}$ springeropen.com 